\title{
IMMUNOSUPPRESSION
}

\section{Peripheral Blood Lymphocyte Phenotype Can Predict Rejection Episodes After Orthotopic Liver Transplantation}

\author{
R. Perdigoto, A. Paiva, A. Freitas, H. Brêda Coimbra, O. Mota, J. Ferrão, L. Tomé, E. Furtado, \\ M.A. Cipriano, B. Geraldes, F.J. Oliveira, and A.L. Furtado
}

$I^{\mathrm{T}}$ T IS WIDELY known that, unlike other transplants, liver allografts behave as an immunologically favored organ and that their outcome is apparently independent of HLA compatibility, showing a special tolerance status and prolonged survival. ${ }^{1}$ It is also known that controling rejection is the most important aspect in the treatment of posttransplant recipients because it determines prognosis. ${ }^{2}$

Changes in T-lymphocyte (Ly) subsets have previously been related to clinical events following liver transplantation and have been of prognostic significance following renal transplantation. ${ }^{3}$ Activation and proliferation of immunocompetent cells is the basic immunologic response to alloantigens, ${ }^{4}$ which could be evaluated by the expression on $\mathrm{T}$ cells of CD25, HLA-DR molecules, and by the loss of the high molecular mass isoform of the CD45 molecule (CD45RA) and the expression of the low molecular mass isoform CD45RO. ${ }^{5}$ Also, the increase of CD8+ Ly coexpressing the CD57 molecule has been found in graft rejection episodes. ${ }^{6}$ Furthermore, Ly adhesion to the endothelium and Ly migration from blood to tissues are critical steps in cellular rejection and depend on cytokine-induced expression of endothelial adhesion molecules. Ly may bind to the endothelium by ICAM-1 (CD54), VCAM-1, and E-selectin-dependent pathways. ${ }^{7}$

The aim of our study was to detect phenotypic changes in peripheral blood Ly of liver transplanted patients, in the absence of bacterial, viral, or fungal infections, in order to predict rejection episodes. For this purpose, we studied patients at pretransplant, twice a week in the first month, and 3, 6, 9, and 12 months after orthotopic liver transplantation (OLT). Immunophenotyping was made by flow cy- tometry and Ly populations and subpopulations were characterized by the following markers: CD3, CD16/CD56, CD19, CD4, CD8, CD4/CD45RA, CD4/CD45RO, CD8/ CD56, CD8/CD57, CD4/CD25, CD8/CD25, CD4/HLADR, CD8/HLA-DR, CD4/CD54, and CD8/CD54.

\section{MATERIALS AND METHODS Patients}

We studied 32 patients immediately before OLT and two times a week in the first month and at the 3rd, 6th, 9th, and 12th month after OLT.

\section{Methods}

Cell immunophenotyping was performed with fluorochrome (order: FITC/PE/TC)-conjugated monoclonal antibodies (MøAbs) by standard three-color immunofluorescence: CD3/CD16+CD56/ CD19; CD45RA/CD45RO/CD4; HLA-DR/CD25/CD4; CD57/ CD56/CD8; HLA-DR/CD25/CD8; and CD4/CD54/CD8. All MøAbs were purchased from Immunotech (Coulter Company, France), except TC-conjugated CD4, CD8, CD19 (Caltag Lab, Calif), and FITC-conjugated CD4 (Serotec Lda, UK).

Briefly, $100 \mu \mathrm{L}$ of EDTA collected venopuncture blood was incubated with an appropriate volume of $\mathrm{M} \varnothing \mathrm{Ab}$ for 10 minutes at

From the Liver Transplant Unit (R.P., O.M., J.F., L.T., E.F., B.G., F.J.O., A.L.F.) and the Department of Pathology, (M.A.C.) Coimbra University Hospital; and from the Histocompatibility Centre of Coimbra (A.P., A.F., H.B.C.), Coimbra, Portugal.

Address reprint requests to Artur Augusto Paiva, Centro Histocompatibilidade do Centro, Faculdade de Medicina, 3001401 Coimbra, Portugal. 
Table 1. Percentage (Mean \pm SD) of B, T, and NK Ly and Subpopulations of T Ly Coexpressing Other Molecules Before and in the First Days After OLT

\begin{tabular}{lcc}
\hline \multicolumn{1}{c}{ Cell Populations } & Before OLT & After OLT \\
\hline CD3 & $76 \pm 9^{*}$ & $50 \pm 15$ \\
CD19 & $14 \pm 7^{*}$ & $40 \pm 15$ \\
CD16/CD56 & $7 \pm 6$ & $7 \pm 5$ \\
CD4 & $51 \pm 10^{*}$ & $32 \pm 11$ \\
CD8 & $24 \pm 6^{*}$ & $18 \pm 6$ \\
CD45RO on CD4+ & $69 \pm 18$ & $62 \pm 18$ \\
CD45RA on CD4+ & $36 \pm 20$ & $40 \pm 20$ \\
CD25 on CD4+ & $15 \pm 9$ & $13 \pm 8$ \\
HLA-DR on CD4+ & $6 \pm 3$ & $7 \pm 4$ \\
CD54 on CD4+ & $13 \pm 6^{*}$ & $26 \pm 12$ \\
CD56 on CD8+ & $19 \pm 17$ & $22 \pm 12$ \\
CD57 on CD8+ & $25 \pm 17$ & $25 \pm 16$ \\
CD25 on CD8+ & $3 \pm 3$ & $3 \pm 2$ \\
HLA-DR on CD8+ & $13 \pm 7$ & $11 \pm 6$ \\
CD54 on CD8+ & $30 \pm 13$ & $34 \pm 16$ \\
\hline
\end{tabular}

*Statistically significant value $(P<.05)$.

room temperature in the dark, followed by red blood cell lysis (Q-PREP, Coulter, Hialeah, Fla). Cells were washed once, resuspended with $0.7 \mathrm{~mL}$ of phosphate buffer saline with $1 \%$ paraformaldehyde, and 10,000 events were analyzed on an Epics XL (Coulter) flow cytometer.

\section{Statistical Analysis}

Statistical analysis was carried out using a standard $t$ test performed by Macintosh (Cupertino, Calif) computer program Statview 512+ (Abacus, Berkeley, Calif).

\section{RESULTS}

Our results in the first days after OLT showed a significant increase in B Ly, a significant decrease in T Ly and their subpopulations CD4 and CD8, and the same percentage of natural killer (NK) cells, which tend to return to pretransplant values around the third week after transplantation. The other Ly subpopulations did not change after OLT, except the T-cell subset CD4+/CD54+ which increased (Table 1).
Immediately before or during rejection episodes, we observed an increase in the percentage of CD8/CD57, CD8/CD56, CD8/CD54, CD8/HLA-DR, CD4/CD45RO, CD4/CD54, and CD4/CD25 double-positive Ly and a decrease in CD4/CD45RA double-positive Ly (Table 2).

\section{DISCUSSION}

Despite the difference in the HLA complex between the donor and the receptor in OLT, the incidence and severity of unresolved acute and chronic rejection episodes are less common than in other solid organ transplants. Liver allograft rejection is mediated by a primary response of $\mathrm{T}$ Ly followed by infiltration of the graft with a mixed inflammatory reaction. ${ }^{8}$

Peripheral blood Ly analysis by flow cytometry, before and after OLT, could be useful in predicting acute rejection episodes, ${ }^{1}$ despite the controversial applications in renal transplantation. ${ }^{9}$ We found major alterations in the percentage of T (CD3, CD4 and CD8) and B Ly after OLT. Almost all subpopulations of CD4 and CD8 Ly remained unchanged, except the subpopulation CD4+/CD54+, which allows detection of percent changes on the other T-cell subsets and correlates them with rejection/infection episodes. Immediately before or during rejection episodes, our results showed in CD4+ Ly an increase in CD45RO, CD54, and CD25, and a decrease in CD45RA doublepositive cells. An increase of CD54, HLA-DR, CD56, and CD57 was observed in CD8+ Ly on rejection episodes. This increase of activated T Ly and CD8 Ly with higher cytolytic potential is in agreement with other studies performed in kidney transplantation. ${ }^{9}$ The immune response to infection could induce similar changes in T-Ly subsets. In order to analyze if the noted changes were able to discriminate between infection or rejection episodes, we treated our results as a ratio of increase (data not shown). Although the number of cases of rejection or infection has been small, the subsets CD4/CD45RO, CD25/CD4, CD56/CD8, CD57/ CD8, and HLA-DR/CD8 presented a higher ratio on rejection than infection, and could be used as a good marker of rejection episodes.

Table 2. Percentage (Mean \pm SD) of T Ly and Subpopulations of CD4 and CD8 Ly Coexpressing Other Molecules on the Last Immunophenotyping Before Rejection Episodes (BRE) and on Rejection Episodes (RE)

\begin{tabular}{|c|c|c|c|c|c|c|c|c|c|c|c|c|c|}
\hline & CD3 & CD4 & CD8 & $\begin{array}{l}\text { CD45RA } \\
\text { on CD4 }\end{array}$ & $\begin{array}{l}\text { CD45RO } \\
\text { on CD4 }\end{array}$ & $\begin{array}{l}\text { CD25 on } \\
\text { CD4 }\end{array}$ & $\begin{array}{l}\text { HLA-DR } \\
\text { on CD4 }\end{array}$ & $\begin{array}{l}\text { CD54 on } \\
\text { CD4 }\end{array}$ & $\begin{array}{l}\text { CD56 on } \\
\text { CD8 }\end{array}$ & $\begin{array}{l}\text { CD57 on } \\
\text { CD8 }\end{array}$ & $\begin{array}{l}\text { CD25 on } \\
\text { CD8 }\end{array}$ & $\begin{array}{l}\text { HLA-DR } \\
\text { on CD8 }\end{array}$ & $\begin{array}{c}\text { CD54 on } \\
\text { CD8 }\end{array}$ \\
\hline JAG BRE & 54 & 39 & 28 & 32 & 63 & 6 & 13 & 25 & 23 & 65 & 1 & 21 & 35 \\
\hline JAG on RE & 79 & 56 & 26 & 37 & 72 & 28 & 15 & 36 & 43 & 75 & 7 & 35 & 55 \\
\hline MFS BRE & 58 & 49 & 12 & 43 & 70 & 12 & 3 & 18 & 21 & 28 & 4 & 14 & 17 \\
\hline MFS on RE & 75 & 55 & 18 & 36 & 77 & 29 & 5 & 33 & 28 & 34 & 8 & 18 & 74 \\
\hline VWF BRE & 95 & 71 & 22 & 51 & 45 & 26 & 2 & 14 & 5 & 12 & 6 & 8 & 27 \\
\hline VWF on RE & 94 & 68 & 23 & 43 & 54 & 27 & 4 & 23 & 9 & 20 & 6 & 14 & 38 \\
\hline JJAS BRE & 61 & 34 & 23 & 47 & 53 & 6 & 6 & - & 10 & 11 & 1 & 6 & - \\
\hline JJAS on RE & 61 & 32 & 22 & 27 & 72 & 12 & 5 & - & 17 & 13 & 1 & 7 & - \\
\hline MHZC BRE & 48 & 37 & 12 & 65 & 34 & 5 & 3 & - & 8 & 9 & 2 & 7 & - \\
\hline MHZC on RE & 55 & 38 & 19 & 51 & 53 & 22 & 7 & - & 25 & 22 & 3 & 19 & - \\
\hline
\end{tabular}

Abbreviations: JAG; MFS; VWF; JJAS; MHZC. 


\section{REFERENCES}

1. García-Alonso AM, Minguela A, Muro M, et al: Hum Immunol 53:64, 1997

2. Sakurai H, Mizuno S, Ohtakara K, et al: Transplant Proc 26:898, 1994

3. Crosbie OM, Norris S, Hegarty JE, et al: Immunol Invest 27:237, 1998

4. Nakajima Y, Hisanaga M, Taki J, et al: Transplantation 55:480, 1993
5. Ibrahim S, Dawson DV, Sanfilippo F: Transplantation 59:724, 1995

6. Lebranchu Y, Kapahi P, Najjar Al, et al: Transplant Proc 26:1873, 1994

7. Hebib C, Leroy E, Rouleau M, et al: Transplant Immunol 6:39, 1998

8. Dollinger MM, Howie SE, Plevris JN, et al: FASEB J 12:939, 1998

9. Oliveira JGG, Ramos JP, Xavier P, et al: Transplantation 64:97, 1997 